\title{
Experimental research of oil-free support systems to predict the high-speed rotor bearing dynamics
}

\author{
Dorota Kozanecka • Zbigniew Kozanecki • \\ Eliza Tkacz • Jakub Łagodziński
}

Received: 20 January 2014 / Revised: 12 February 2014 / Accepted: 27 February 2014 / Published online: 19 March 2014

(C) The Author(s) 2014. This article is published with open access at Springerlink.com

\begin{abstract}
In modern dissipated power generation systems, microturbines of power output ranging between 1 and $20 \mathrm{~kW}$ are applied. The development of a reliable bearing technology for high-speed small turbomachinery could be essential to these power-generating devices. In order to introduce this technology to common use, the optimal design selection from the viewpoint of machine reliability must be conducted. Therefore, one should analyze thoroughly the dynamics of the rotor-bearing-casing system in the whole operating range of the machine. The rotating system presented in the paper is supported in oil-free, airfoil bearings. Compliant surface foil gas bearings are a class of hydrodynamic bearings that use the ambient gas as their working fluid and thus require no dedicated lubrication systems, which makes their design much simpler. The article presents the development and experimental verification of a theoretical numerical model of the foil bearing for analysis of its dynamic characteristics (a sum of properties: two elastic elements connected in a series and their relative motion, friction that is related to this motion with respect to the elastic and cylindrical foils subjected to deformation) that will be an useable part of the study referring to numerical analyses oriented on developing a model of the high-speed rotor supported in bump-foil bearings.
\end{abstract}

\footnotetext{
D. Kozanecka · Z. Kozanecki · E. Tkacz · J. Łagodziński $(\bowtie)$ Institute of Turbomachinery, Lodz University of Technology, 219/223 Wólczańska Street, 90-924 Lodz, Poland

e-mail: jakub.lagodzinski@p.lodz.pl

D. Kozanecka

e-mail: dorota.kozanecka@p.lodz.pl

Z. Kozanecki

e-mail: zbigniew.kozanecki@p.lodz.pl

E. Tkacz

e-mail: eliza.tkacz@p.lodz.pl
}

Keywords Oil-free support · High-speed turbomachinery · Gas bearings $\cdot$ Foil bearings

\section{Introduction}

The micro-power system, with the power output ranging between 0.05 and $20 \mathrm{~kW}$, is based on the Brayton or Rankine cycle and consists of a turbine (gas turbine) and a generator. The system requires high revolutions to generate sufficient power in the small-size turbine (microturbine). The term "microturbine" is often used for turbine systems characterized by the power output from a few watts to hundreds of $\mathrm{kW}$. In this field, we consider a microturbine to produce electricity in this power range at the speed of approximately 10,000-100,000 rpm.

The existing conventional oil-lubricated slide or ball bearings reveal performance limits at these revolutions, especially when a power or stability loss of the bearing is taken into account. Furthermore, more and more common applications of non-conventional materials in the machine design provide opportunities for considering an idea of applying the working medium used in a small high-speed turbomachine as a lubricating medium for its bearings very realistic.

In machines in which a low-viscosity working gas or liquid is used, an application of bearings lubricated by that working medium makes it possible:

- to increase the total efficiency of the machine, which is attained by decreasing friction losses in the bearings and by eliminating the oil system and seals connected with it;

- to simplify the design of the shaft and to reduce its length;

- to maintain absolute purity of the working medium; 
Fig. 1 Aerodynamic foil bearing developed for the experimental needs

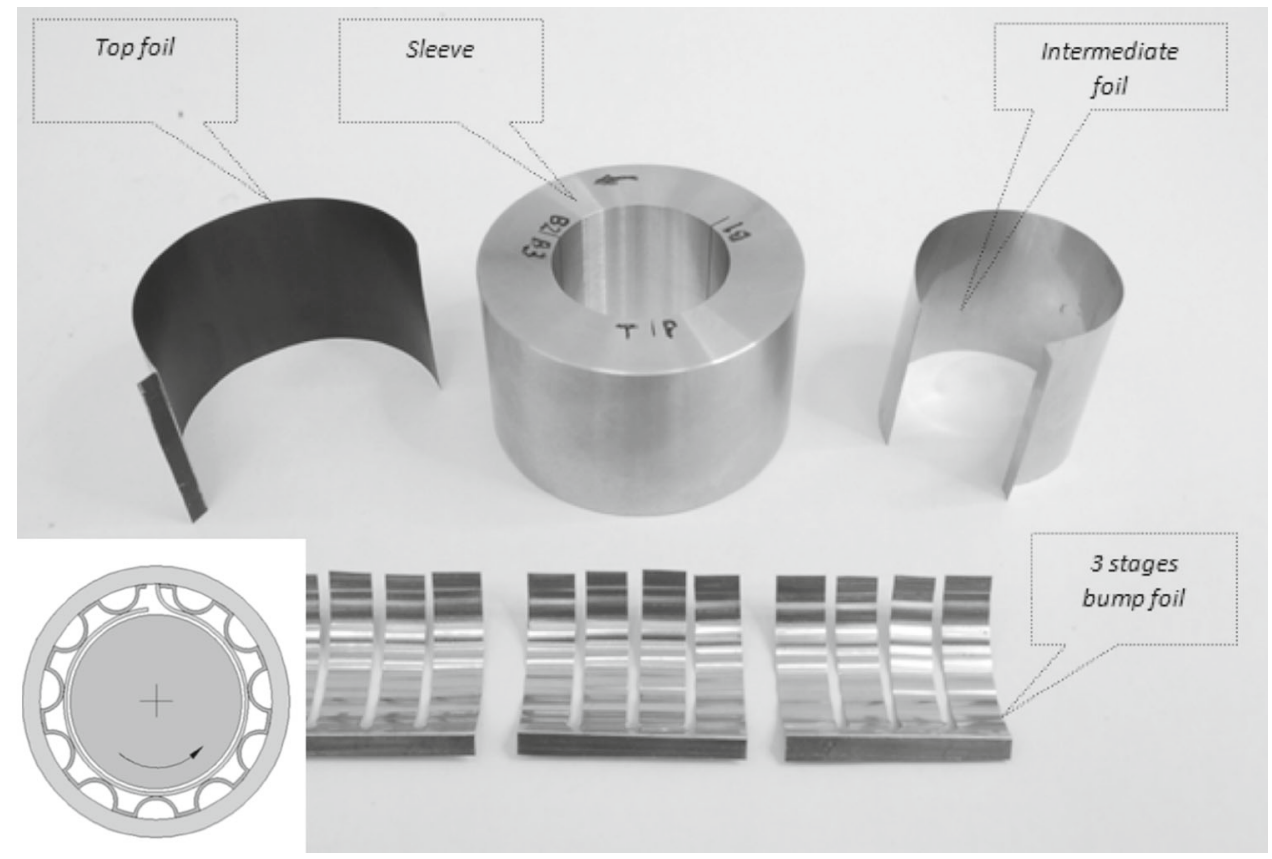

- to build a "hermetic" machine without a rotating shaft end protruding outside the casing, to eliminate the mechanical gear and "working medium - atmosphere" seals in an electric generator or motor integrated with the shaft.

While studying possible applications of non-conventional lubricating media, one should analyze thoroughly the dynamics of the "rotor-bearing-casing" system within the whole range of machine operation [4].

\section{Gas bearing technology}

The compressibility of gas is an important factor and it has to be included in the analysis of various forms of gas bearingsboth aerodynamic and aerostatic (self-acting and externally pressurized). The advantages over liquid-lubricated bearings are well known: cleanliness, stability of the lubricant, very low friction (low viscosity). The main disadvantages of gaslubricated bearings are recognized as resulting from low viscosity of the gas: reduced unit load carrying capacity, especially in aerodynamic (self-acting) bearings, closer control of manufacturing tolerances.

Gas bearings are to be met in a variety of applications from small laboratory devices to special high-speed devices. Their development is connected with the fact that they can be often used where the application of well-known traditional design solutions of bearings is troublesome (i.e., high-speed lowor high-temperature applications-cryogenic expanders or gas turbines). Actually, three kinds of gas bearing systems are developed simultaneously for the micro-turbomachine needs: aerostatic gas bearings, aerodynamic compliant surface ("bump foil") bearings and aerodynamic "tilting pad" gas bearings.

Despite of the good rotordynamic characteristics of the machine, the main problem connected to the aerostatic bearing application is additional energy needed to pressurize the supply pressure orifices spaced uniformly in two ranges of the bearing sleeve [1]. From the viewpoint of energy consumption, one should analyze thoroughly the total mass flow of the incoming pressurized vapor through the aerostatic bearing system. The bearing mass flow reduces the total mass flow of the turbine and, consequently, decreases the efficiency of the machine. Consequently, from this point of view, aerodynamic compliant surface ("bump foil") bearings lubricated by ambient gas are much more interesting.

\subsection{Aerodynamic foil bearings}

A structure of the experimental foil bearing is shown in Fig. 1. The uniqueness of foil bearing operation results from the fact that the top foil is clenched during the bearing operation on the rotating journal by means of the elastic bump foil. The aerodynamic film geometry of very low thickness, theoretically close to the cylindrical one, is generated by the viscosity effects. A great advantage of aerodynamic bearings is that they require no external pressurization system for the working fluid.

An important problem of the aerodynamic gas bearing application is connected to the start-up and the shut-down in contact with the shaft surface and thus: 


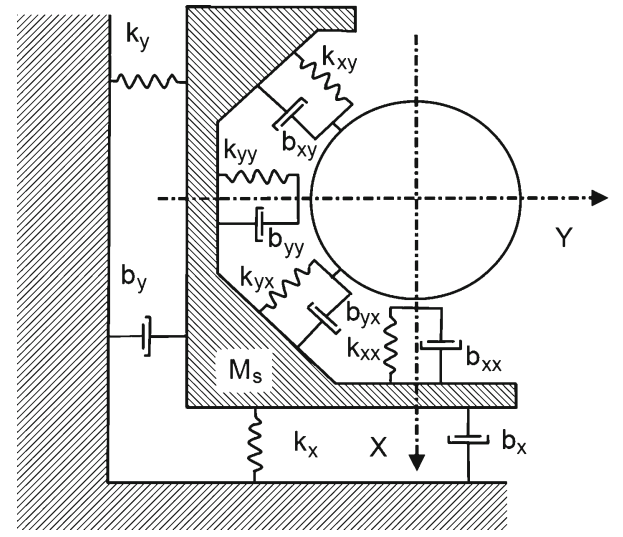

Fig. 2 Mathematical model of the foil bearing

- a high drive moment for the start-up (disputable from the viewpoint of the turbine mechanical characteristics) is needed,

- there is a limited number of start-up/shut-down cycles (wear).

Furthermore, the theoretical analysis of gas foil bearings is difficult due to an interaction between the gas film pressure and the complicated deflection of the top foil and the underlying bump strip support structure.

\subsection{Computational model of foil bearing dynamics}

Dynamic properties of aerodynamic gas bearings, according to the linear theory, are usually represented by a set of eight coupled dynamic coefficients $\left(k_{i j}, b_{i j}\right)$, linearized around the static equilibrium position of the bearing (see Fig. 2). It is necessary, however, to limit the scale of the excitation forces in order to fulfill the basic condition of small displacements around the equilibrium point. It allows one to linearize the forces in the bearing film and to determine the equivalent values of coupled bearing stiffness and damping coefficients.

It should be highlighted that the lack of precise criteria, which allow one to determine the applicability range of the linear method, often leads to serious errors in the dynamic analysis of the rotor-bearings system.

\subsection{Numerical simulations of the gas film in the foil bearing}

In order to carry out an analysis of the bearing dynamics, it is necessary to find the static equilibrium position for the journal. In such a position of the shaft, the force resulting from a geometric sum of the vectors of the forces in the film balances the external static loading of the journal.

An application in Fortran was created in order to analyze the gas film of the foil bearing and find the equilibrium position of the shaft for the applied static force. We find the pressure distribution from the Reynolds equation for compressible flows [2] by means of numerical computations. The results from the numerical simulations are shown in Fig. 3, where the pressure distribution at the top foil was found for various film geometries, including the cylindrical one.

The bearing static equilibrium position determines the initial conditions for an analysis of the bearing dynamics. Then,
Fig. 3 Pressure distribution for a cylindrical film thickness

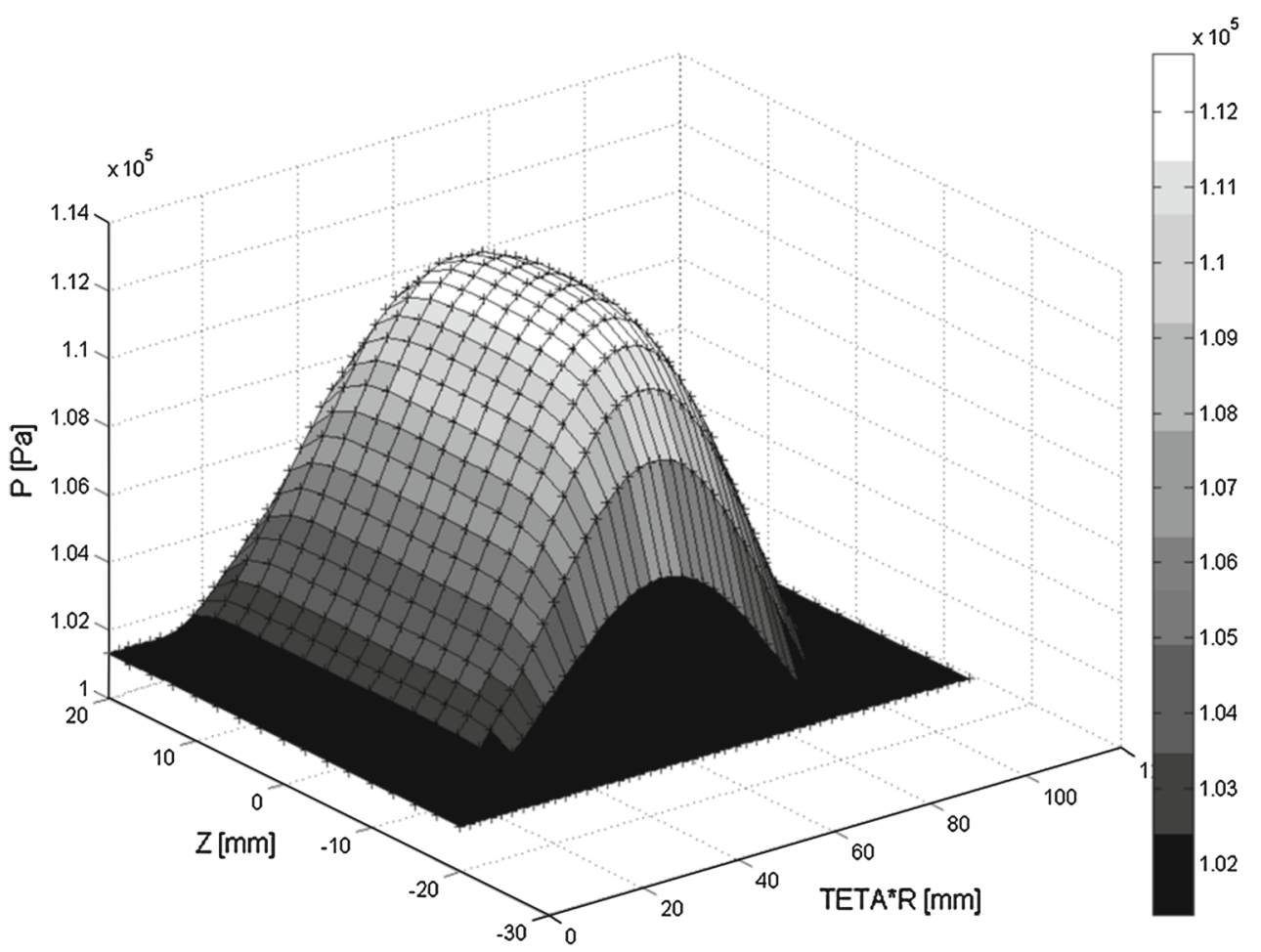



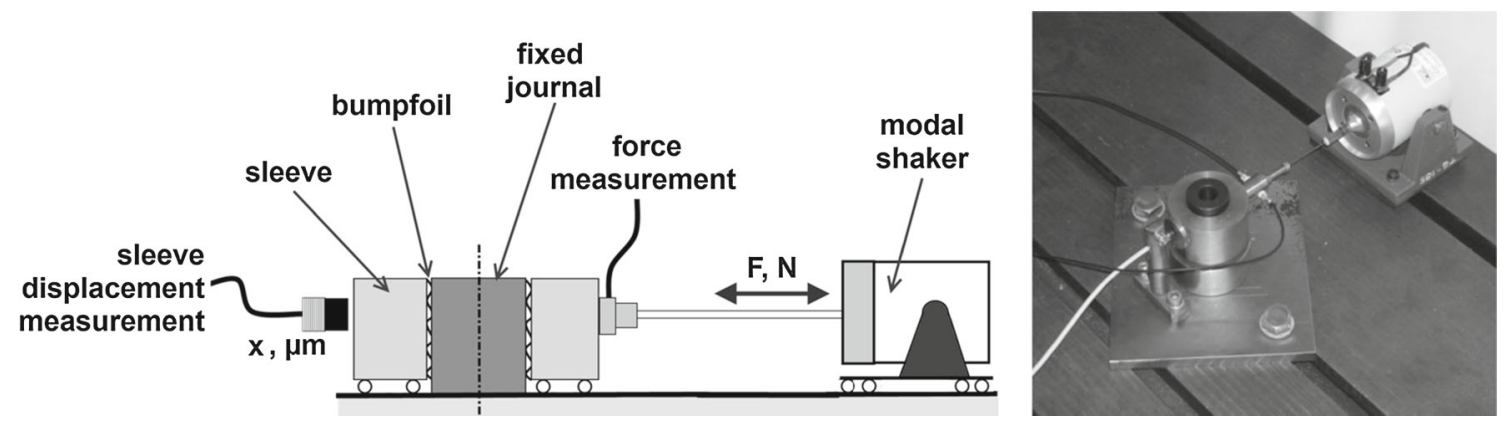

Fig. 4 Functional diagram and a photo of the test rig built for the experimental identification of dynamic properties of the Third+ generation foil bearing support structure

we introduce the position perturbation and the velocity perturbation, so that the Taylor equation in the matrix form for the gas film reaction forces can be formulated [2], whence stiffness and damping coefficients are defined as follows

$$
\begin{aligned}
& \left.\frac{\partial F_{x}}{\partial x}\right|_{0}=K_{x x},\left.\quad \frac{\partial F_{x}}{\partial y}\right|_{0}=K_{x y},\left.\frac{\partial F_{y}}{\partial x}\right|_{0}=K_{y x}, \\
& \left.\frac{\partial F_{y}}{\partial y}\right|_{0}=K_{y y}, \\
& \left.\frac{\partial F_{x}}{\partial \dot{x}}\right|_{0}=B_{x x},\left.\quad \frac{\partial F_{x}}{\partial \dot{y}}\right|_{0}=B_{x y},\left.\frac{\partial F_{y}}{\partial \dot{x}}\right|_{0}=B_{y x}, \\
& \left.\frac{\partial F_{y}}{\partial \dot{y}}\right|_{0}=B_{y y} .
\end{aligned}
$$

The analysis of gas foil bearings is difficult because of an interaction between the gas film pressure and the complicated deflection of the top foil and the underlying bump strip support structure. In order to calculate dynamic properties of the whole bearing and thus to calculate the rotor dynamics, we have to take into account elastic and damping properties of both the foil and gas film.

\subsection{Dynamic properties of the compliant structure of the bump foil bearing}

At present, the investigations connected to the optimal design of the bump foil bearing are conducted in two main directions:

- to select the optimum pair of materials (a journal material and a foil coating material) that will ensure its sufficient durability under different machine operating conditions (start-up, nominal speed, shut-down),

- to find the optimum design of the bump foil thrust and radial bearings for the considered application and to determine reliable methodology of prediction of dynamic properties of the compliant surface bump foil bearing.

The results of the investigations related to the material selection are described in [3].
In Fig. 2, where a simplified physical model of the bump foil bearing is shown, static and dynamic characteristics of the foil bearing are the result of elastic combined properties of the two elements serially connected. One of them is a thin gas film of very small thickness and relatively high static and dynamic stiffness $\left(k_{i j}\right)$. The second elastic element is a pre-tensioned bump foil spring $\left(k_{i}\right)$.

It should be noticed that depending on the bump foil pretension, a stiff gas film appears at a few to over a dozen krpm. Above this speed limit, a continuous gas film occurs, and the foil bearing operates properly when the rotating journal loses contact with the top foil. The accepted physical model of the start-up of aerodynamic foil bearings allows one to formulate the following statements:

- for the journal rotational speed below $n_{\text {lim }}$, where $n_{\text {lim }}$ denotes the rotational speed at which the continuous gas film appears, it can be assumed that the dynamic properties of the bearing depend on the stiffness of bump foil springs, because the gas film equivalent stiffness coefficient is large in comparison to the foil structure one $\left(K_{G} \gg K_{F}\right)$,

- at the journal rotational speed above $n_{\text {lim }}$, theoretically, the dynamic properties of the support system depend on the combined stiffness of the two elements serially connected.

The complexity of the analysis of the foil bearing theoretical model is caused by friction between the bump foil and the sleeve and between both foils. The friction comes from a relative motion of the foils and a relative motion of the bump foil and the sleeve. This physical phenomenon results in highly nonlinear dynamic properties of the foil bearing support system.

Some experimental attempts were made to identify these properties and a foil bearing test rig (see Fig. 4) was built for this purpose. The test rig consisted of a fixed journal, frictionlessly supported sleeve and a modal shaker. The shaker excited the sleeve with a sinusoidal waveform force. It simu- 




Fig. 5 Image response as a hysteresis loop associated with friction and elasticity of the compliant foil bearing assembly

lated real synchronous excitation caused by rotor unbalance. During the experiment, the excitation force and the sleeve displacement were measured.

A typical response of the shaking system is a hysteresis loop, presented in Fig. 5. From this image, one can obtain the bump spring overall stiffness $k$ and an area of the hysteresis loop $W$ that represents the energy dissipated in a single motion cycle. The energy dissipation is related to the friction between the bearing foils and can be estimated from Eq. 1:

$W=\oint F d x=\pi \omega C_{e q} X^{2}$

where $W$-area of the hysteresis loop, $X$-vibration amplitude, $\omega$-circular frequency and $C_{e q}$-equivalent damping coefficient.

Transforming Eq. 1, we obtain $C_{e q}$ :

$c_{e q}=\frac{W}{\pi \cdot \omega \cdot X^{2}}$

Fig. 6 Exemplary hysteresis loops obtained from the measurements at different excitation frequencies

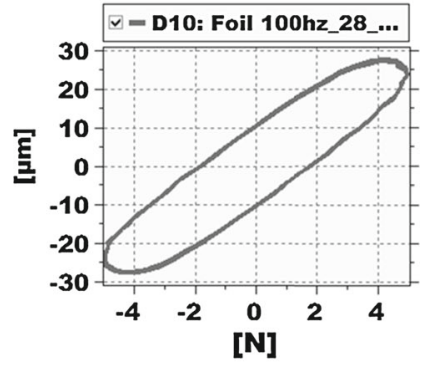

In the experiment, the bearing displacement amplitude was measured at the constant excitation amplitude of $10 \mathrm{~N}$ $\mathrm{p}-\mathrm{p}$, for the excitation frequency ranging from 80 to $600 \mathrm{~Hz}$. For these test conditions, a few obtained hysteresis loops are presented in Fig. 6.

The obtained hysteresis loops allowed for identification of the experimental stiffness $\left(K_{F}\right)$ and damping $\left(C_{F}\right)$ values of the compliant foil bearings built for the test model of the rotating system (see Fig. 7).

As can be observed (see Fig. 7), the bearing stiffness increases exponentially with the excitation frequency. The damping coefficient estimated from Eq. 2 develops fully at $200 \mathrm{~Hz}$ and weakens noticeably above $400 \mathrm{~Hz}$. The damping characteristics of the foil bearing is highly nonlinear.

\section{Test rig}

In further investigations on foil bearings, it was decided to build a complex test bench, which allows one to test a prototype set of the journal-thrust bearings under real static and dynamic loads, which occur under motor operation and loading forces associated with operation of the radial flow compressor impeller.

The Fig. 8 shows a structure of the microcompressor test rig, which was built in order to assess the suitability of journal-thrust foil bearings. This installation is a geometric representation of the turbogenerator prototype built for the organic Rankine cycle (ORC) needs [1]. The machine works successfully with aerostatic gas bearings and it is a target


Fig. 7 Stiffness and equivalent damping coefficients of the compliant foil bearing support system versus excitation frequency 
Fig. 8 Microcompressor supported in the compliant foil bearings
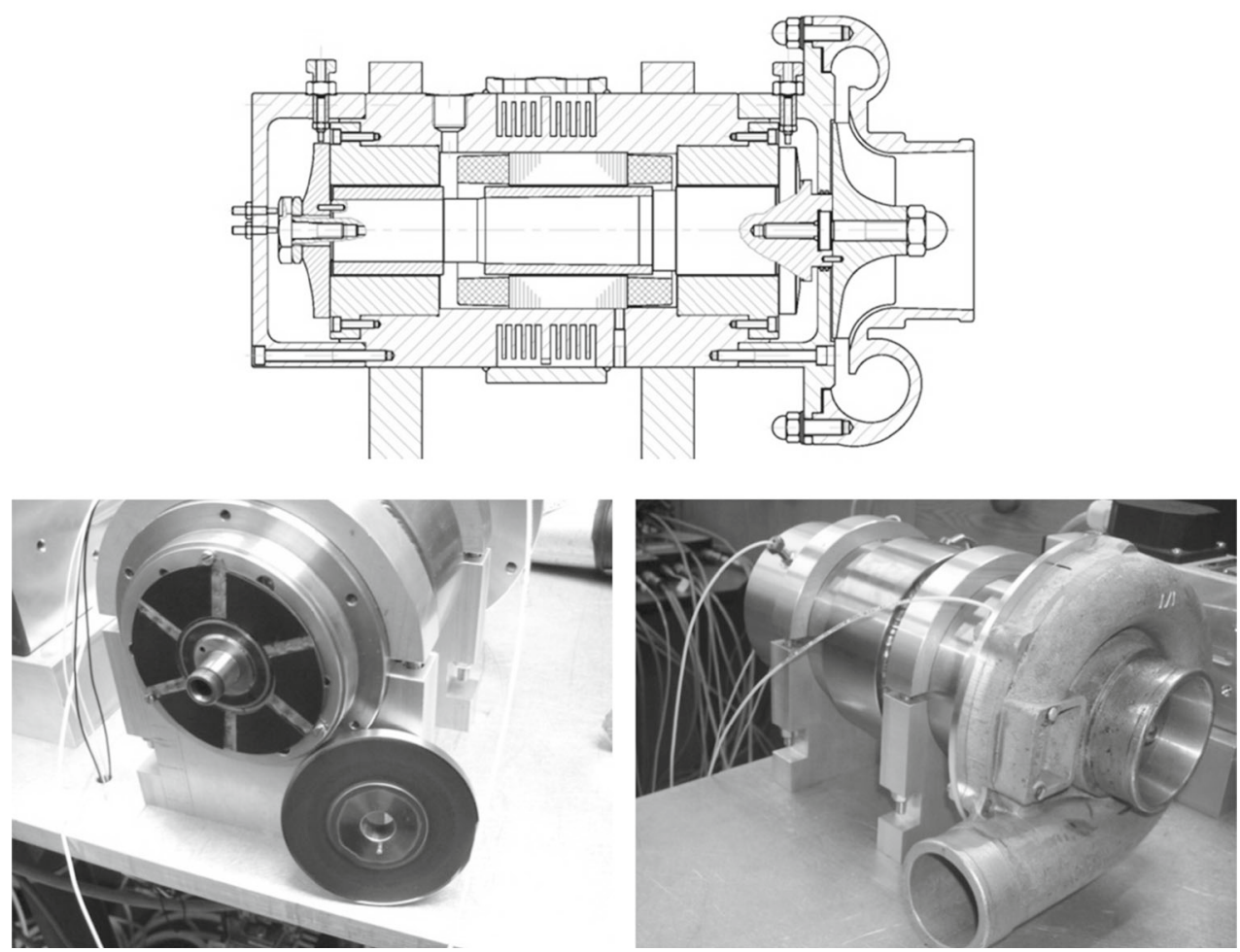

BODE PLOT

COMPANY: IMP PL

MACHINE TRAIN: dmuchawa foliowe

POINT: Channel DE $\times 1 \quad \quad \angle 45^{\circ}$ Right
POINT: Channel DE $\times 1 \quad \quad \angle 45^{\circ}$ Right
MACHINE: Compressor foil brg

From 15MAR2013 10:52:25.1 To 15MAR2013 10:53:36.5 Startup
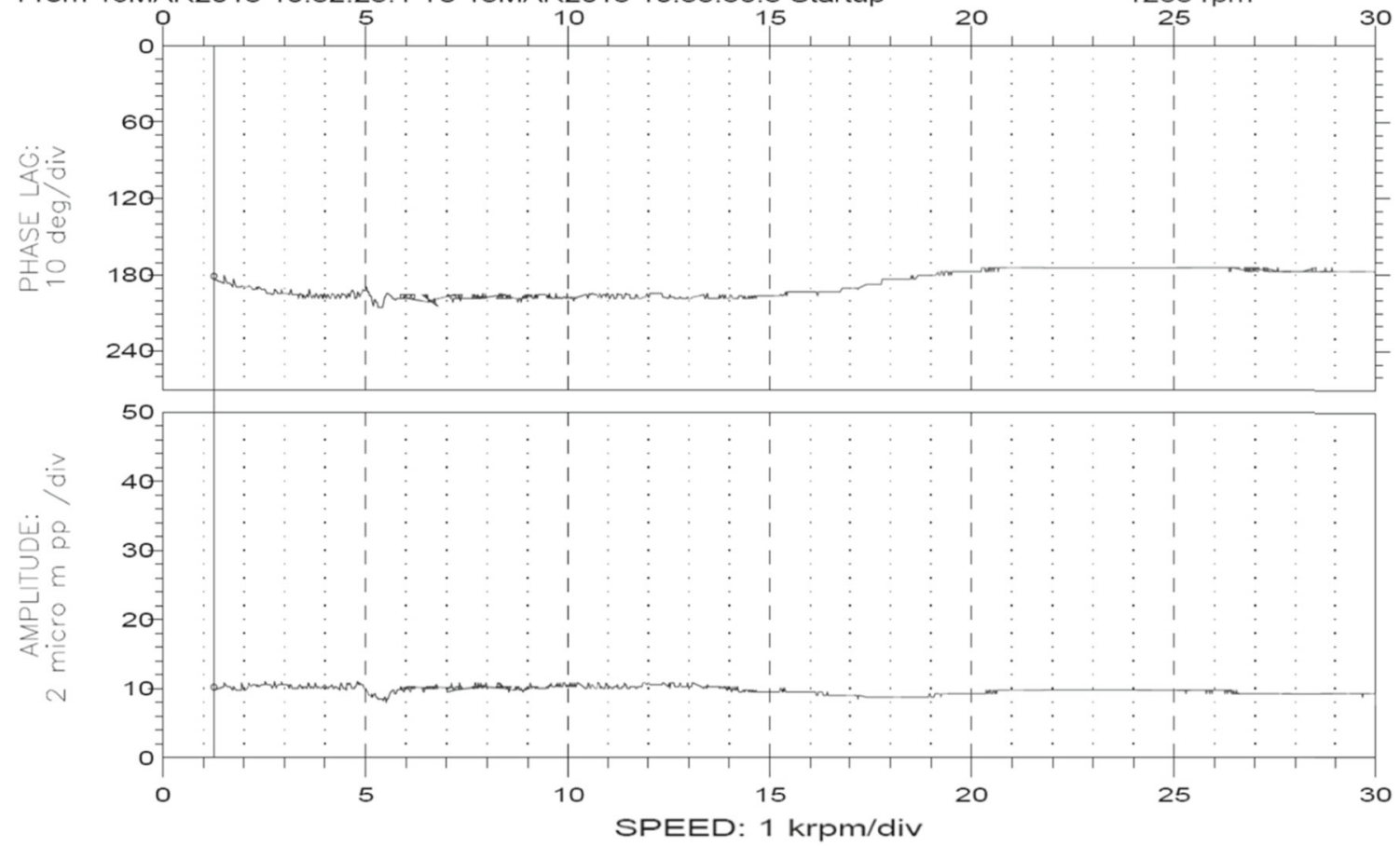

Fig. 9 Relative vibrations of the rotational system measured on the microcompressor test rig during the start-up 
foil structure_Channel DE X1

Station: 13 , Sub-Station: 1
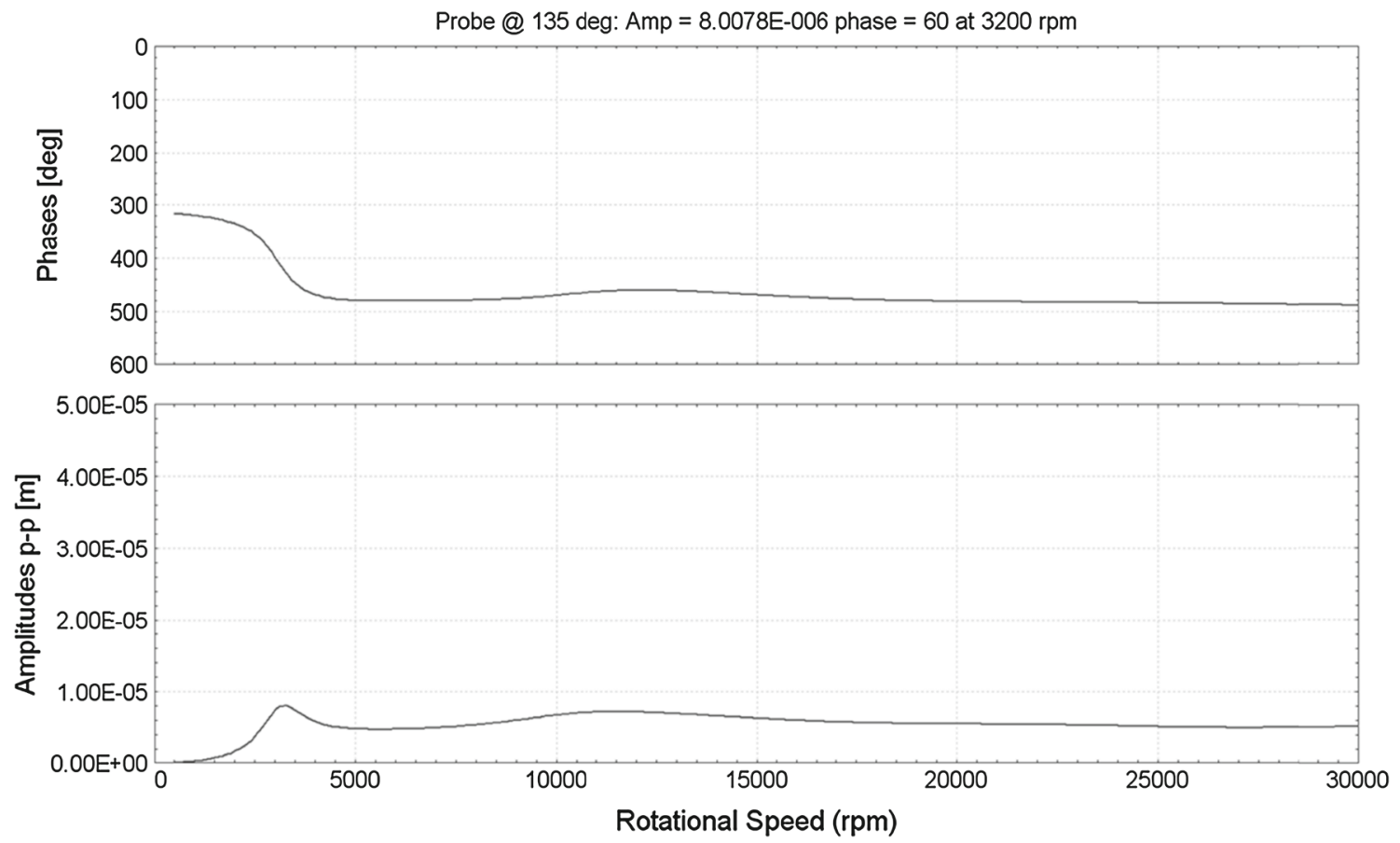

Fig. 10 Results from the computational model of the rotor dynamics-case A

foil structure + gas bearing_Channel DE X1

Station: 13, Sub-Station: 1

Probe @ 135 deg: Amp $=7.9476 \mathrm{E}-006$ phase $=62$ at $3200 \mathrm{rpm}$
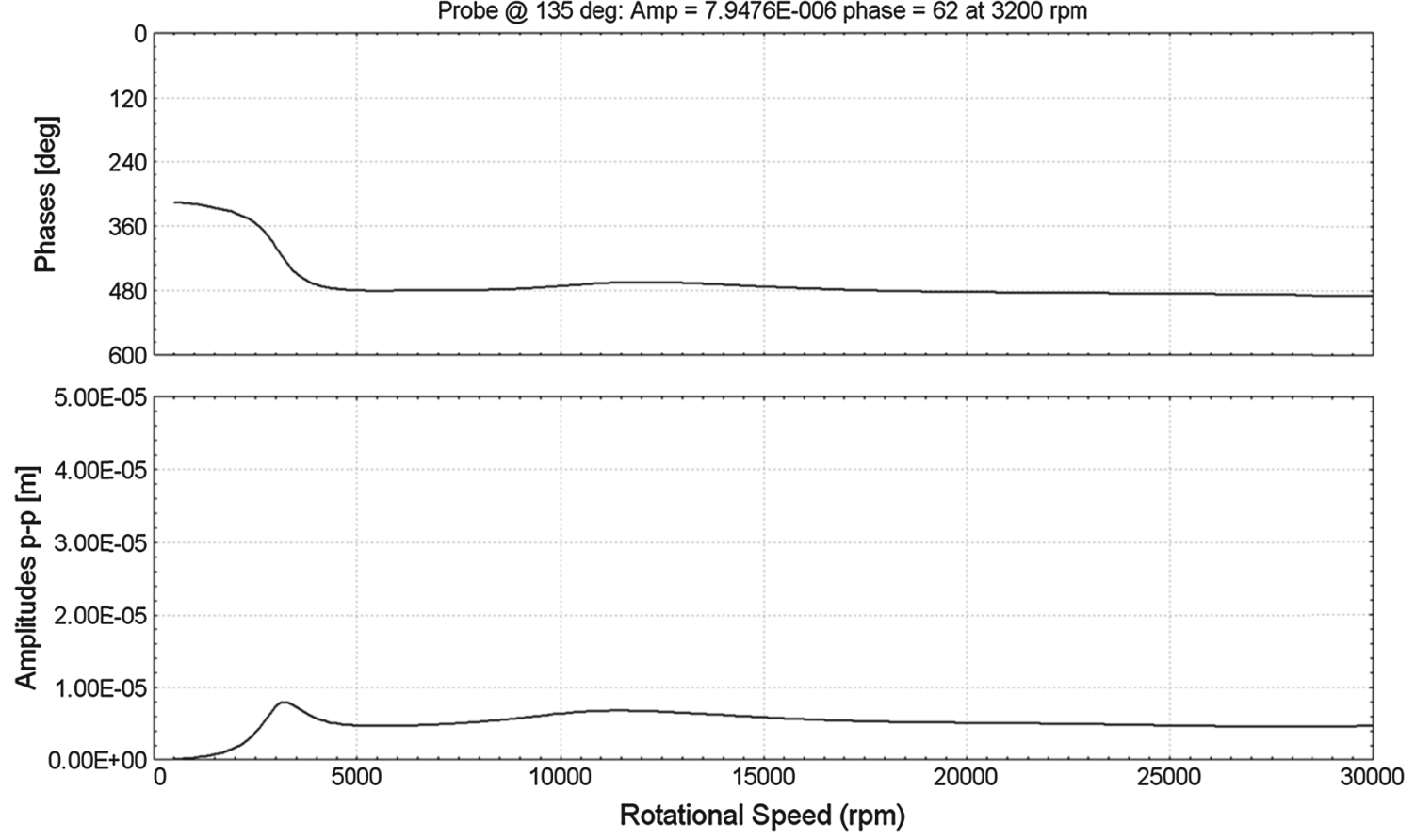

Fig. 11 Results from the computational model of the rotor dynamics-case B 
application for foil bearings, for the sake of efficiency and reliability. Main differences between the microcompressor test rig and the ORC turbogenerator one are:

- aerostatic gas bearings were replaced by journal-thrust foil bearings, which implies absence of supply channels,

- installation in the machine body of the relative vibration measurement system,

- the radial turbine was replaced by a radial compressor, which implies an appearance of significant axial forces,

- the generator worked in the motor mode and it was powered by a high frequency inverter.

The preliminary experiments of the microcompressor runup and operation at the nominal speed have confirmed the earlier assumptions so far. The rotor operation at the nominal speed is stable, with a minor level of vibrations.

3.1 Exemplary computational and experimental results of the microcompressor supported in radial-thrust foil bearings

In Fig. 9, characterics of relative vibrations (Bode plots) during the start-up of the microcompressor are shown. For rotational speeds in the range of $n \leq n_{\text {lim }}$, where $n_{\text {lim }}=$ $15,000 \mathrm{rpm}$, dry friction occurs, hence the bearing system is not working properly yet. At the same time, the Bode plot is compared with the results from the computational model of the rotor dynamics. Case A takes into account only the dynamic characteristics of the elastic structure in foil bearings (see Fig. 10). Case B takes into account dynamic characteristics of both-the elastic structure and the gas film (see Fig. 11).

Small changes in the phase of relative vibrations for rotational speeds $n \geq n_{\text {lim }}$, where the gas film is fully developed, are connected with its stiffening while the speed and the relative motion of the elastic foil system increase.

\section{Conclusions}

- During the experimental tests, some relevant information about the global stiffness and damping coefficients of the elastic foil bearing structure was collected. This will enable the development of a reliable model of the compliant bearing support with linear dynamic coefficients.

- The motion of movable, but non-rotating parts of variable-geometry bearings is inevitably connected with friction. It is evident that the nature of elastically supported bush motion can dramatically affect dynamic characteristics of the bearing. Therefore, nonlinear modeling of dynamic properties, including design characteristics of the support and the generated friction forces, becomes indispensable in high-speed foil bearing applications.

- From the operating point of view, the tested foil bearing has an optimal damping characteristics. In the useful range of rotational speed, the compliant structure is characterized by a significant damping value, due to the full-fledged phenomenon of dry friction between the top foil and the bump foil.

- The preliminary experiments of the micro-compressor run-up and operation at the nominal speed have confirmed the earlier assumptions so far.

Open Access This article is distributed under the terms of the Creative Commons Attribution License which permits any use, distribution, and reproduction in any medium, provided the original author(s) and the source are credited.

\section{References}

1. Tkacz E, Kozanecka D, Kozanecki Z, Miazga K (2011) Investigations of oil-free support systems to improve the reliability of ORC hermetic high-speed turbomachinery. Mech Mech Eng 15(3):355365

2. Kozanecki Z, Tkacz E, Łagodziński J, Miazga K (2012) Oil-free bearings for hermetic high-speed turbomachinery. In: Conference proceedings VETOMAC-VIII, ISBN 978-83-88237-61-4, Gdańsk

3. Kozanecki Z, Kozanecka D (2010) Theoretical and experimental investigations of oil-free support systems to improve the reliability of industrial turbomachinery. In: The 8th IFTOMM international conference on rotor dynamics, 12-15 September 2010. KIST, Seoul, Korea, pp 686-692

4. Tkacz E, Kozanecka D, Kozanecki Z, Łagodzinski J (2013) Theoretical and experimental investigations of oil-free support systems to predict high-speed rotor bearing dynamics. In: Dimitrovová Z, de Almeida JR, Gonçalves R (eds) Proceedings of the 11th international conference on vibration problems (ICOVP-2013), Lisbon, Portugal, 9-12 September 2013. AMPTAC, ISBN 978-989-96264$4-7$, abstract p 357, article 10 pages 